\section{BMJ Open \\ Respiratory \\ Research}

\title{
Prevalence of gastrointestinal dysmotility and complications detected by abdominal plain films after lung transplantation: a single-centre cohort study
}

\author{
Henriette Heinrich, ${ }^{1}$ Anne Neuenschwander, ${ }^{2}$ Stefan Russmann, ${ }^{3}$ \\ Benjamin Misselwitz, ${ }^{1}$ Christian Benden, ${ }^{2}$ Macé M Schuurmans ${ }^{2}$
}

To cite: Heinrich $\mathrm{H}$, Neuenschwander A, Russmann $\mathrm{S}$, et al. Prevalence of gastrointestinal dysmotility and complications detected by abdominal plain films after lung transplantation: a singlecentre cohort study. BMJ Open Resp Res 2016;3: e000162. doi:10.1136/ bmjresp-2016-000162

Received 6 September 2016 Accepted 18 November 2016

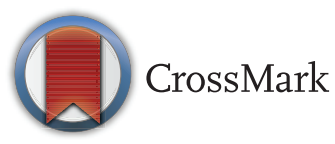

${ }^{1}$ Division of Gastroenterology and Hepatology, University Hospital Zurich, University of Zurich, Switzerland

${ }^{2}$ Division of Pulmonary Medicine, University Hospital Zurich, Zurich, Switzerland ${ }^{3}$ Division of Clinical Pharmacology and Toxicology, University Hospital Zurich, Zurich, Switzerland

Correspondence to Dr Macé M Schuurmans; mace.schuurmans@usz.ch

\section{ABSTRACT}

Introduction and Aims: Gastrointestinal (Gl) complications such as gastric retention (GR) and constipation are common after lung transplantation (LT). Abdominal plain films (APFs) are a low-cost diagnostic tool to detect impaired Gl function. The goal of our study was to assess the prevalence of $\mathrm{Gl}$ pathology seen on APF in lung transplant recipients (LTRs) and to identify associated risk factors.

Methods: Retrospective analysis of consecutive LTRs followed up between 2001 and 2013. Demographic, radiographic and clinical data were assessed.

Results: 198 patients were included in the study, 166 thereof had more than 1 APF with a mean number of 5 APFs per patient. 163 patients had a detectable radiographic pathology on APF. The proportion of LTR with GR was highest among cystic fibrosis patients $(48.5 \%)$. Multivariate regression analysis showed a significant association of diabetes with GR with a trend for age and use of opiates as risk factors. Similarly, female sex, advanced age and diabetes showed a trend to be associated with lower Gl tract complications. Almost all patients had suffered from at least 1 episode of lower Gl dysmotility during a median follow-up of 5.7 years. No clear correlation between Gl events and the development of chronic lung allograft dysfunction could be identified.

Conclusions: We found a statistically significant association of diabetes with GR and a progressive increase in the prevalence of GR over time after LT. Lower GI complications affected $>80 \%$ of LTR and increased over time. Future studies correlating $\mathrm{Gl}$ transit with APF findings are needed.

\section{INTRODUCTION}

Lung transplantation (LT) is performed in patients with non-malignant end-stage lung diseases. Gastrointestinal (GI) complications impact considerably on allograft and patient survival and are frequently encountered in

\section{KEY MESSAGES}

- Upper and Lower Gl dysmotility is common among lung transplant recipients.

- Abdominal plain films are a simple intial test to assess the presence of dysmotility in these patients.

- Gastric retention is common in patients after lung transplantation and is associated with diabetes mellitus.

this population. ${ }^{1-4}$ The underlying disease leading to LT and pre-existing comorbidities contribute to the development of GI complications after transplantation.

GI complications are frequent in lung transplant recipients (LTRs) and are related to LT surgery itself with possible vagal nerve damage and altered diaphragmatic function, immunosuppressive and other medications used, which may affect intestinal motility and content and consistency (ie, bacterial flora altered by prophylactic antibiotics or medication with laxative characteristics). Decreased GI motility of the upper and lower GI tract is frequently noted, despite the routine use of prokinetic and laxative medication in LTR. Among the most frequently observed complications are constipation, gastric retention (GR), intestinal obstruction or perforation. ${ }^{56}$

Often abdominal complications initially present with only minor or even absent symptoms and signs, so that laboratory investigations and imaging are the basis of diagnosis and treatment. ${ }^{5}$ Abdominal radiography usually as abdominal plain film (APF) is a simple, readily available diagnostic tool to visualise consequences of delayed GI transit due to GR, intestinal obstruction and constipation. It may also detect free air in the 
abdominal cavity due to perforation. Diagnosis of these conditions should trigger prompt further diagnostic steps and guide treatment, since these complications have significant impact on necessary medical or surgical treatment and patient survival. ${ }^{78}$

The objective of this study was to assess the prevalence of radiological evidence of GR, intestinal and colonic dysmotility detected on APF obtained for unclear abdominal symptoms in LTRs. Furthermore, we aimed to evaluate risk factors for GI complications as well as the association of GI complications with the development of chronic lung allograft dysfunction (CLAD), in particular its most frequent form bronchiolitis obliterans syndrome (BOS) and patient survival.

\section{METHODS}

We conducted a retrospective chart review and analyses of the cohort of all LTRs at the University Hospital Zurich. Patients who died within the first month of transplantation and patients who died before or in 2001 were excluded from analysis. Furthermore, patients were excluded from analysis if they refused retrospective data analysis.

LT is performed only at two centres in Switzerland, Lausanne and Zurich. Patients receiving LT in Zurich are treated according to our previously published protocols, which include early postoperative and ongoing prokinetic and laxative treatment as well as proton pump inhibitors without prior motility testing and regular 24-hour $\mathrm{pH}$ studies as the prevalence of gastrooesophageal reflux and impaired GI motility is deemed to be high after $\mathrm{LT}^{5}$ BOS is regularly treated with macrolides, which has additional prokinetic effects. Additionally, annual abdominal ultrasound scans are performed in patients with $\mathrm{CF}$ before and after transplantation.

For a follow-up period from January 2001 to November 2013, we documented and analysed comprehensive data on patient demographics, circumstances of LT and medication as well as frequency and results of abdominal radiographs taken irrespective of LT date. Two observers (AN, HH) reviewed all APFs and verified findings of the radiological report. In cases of discordance between the radiology report and the viewer's assessment of APF, a third viewer was involved (MMS).

Coprostasis was documented when faecal loading in either part of the colon without signs of obstruction was present. Small bowel obstruction was defined as the presence of dilated small bowel loops of more than $3 \mathrm{~cm}$ in diameter in the centre of the abdomen. Large bowel obstruction was documented when dilation of the colon to more than $6 \mathrm{~cm}$ or more than $9 \mathrm{~cm}$ for the caecum was seen on APF. Diffuse bowel obstruction was noted when dilation of bowel loops could not be assigned to the colon or small bowel. The presence of subdiapragmatic air was regarded as a sign of perforation. ${ }^{9-11}$

GR was defined as an upper GI event. ${ }^{9}$ Lower GI events were defined as the presence of coprostasis, small and/or large bowel obstruction, diffuse bowel obstruction and perforation on APF. Evidence of complications like ileus and necessary surgery were documented from the electronic records.

Furthermore, lung function before, at the time of the respective GI event and 6 months thereafter was evaluated in order to assess the presence or progression of CLAD/BOS in the time after the event. ${ }^{12}$

Statistical analyses were performed using STATA V.13.1 (StataCorp, College Station, Texas, USA). Data management and descriptive statistics included presentation of results in tables and graphs as appropriate. Risk factors for the development of GP were calculated as unadjusted univariate relative risks and as adjusted ORs using multivariate logistic regression. Time-to-event analyses were performed using the Kaplan-Meier curves.

Permission for retrospective data analysis was obtained from the Local Ethics Committee (KEK-ZH-Nr.2015-0162). All patients had provided written informed consent for research at the time of LT.

\section{RESULTS}

We included 198 patients who had received lung transplants at our centre into the study cohort and followed those between January 2001 and November 2013. Demographics and characteristics of the study population are presented in table 1 . All 198 LTR contributed to a combined total follow-up time of 1274 patient-years with a median follow-up time of 5.7 years (range 74 days-12.9 years). Cystic fibrosis was the most frequent indication for LT accounting for $39.4 \%$ of the study cohort. There were 963 APFs taken during follow-up for the whole cohort, with a great variation in frequency as shown in table 1.

Thirty-three patients (16.7\%) had no APF at all and $33(16.7 \%)$ had on average more than 2 APF per patient-year of follow-up. During follow-up, 26 patients $(13.1 \%)$ died; of these, the cause of death was related to a GI complication in only 2 patients with GI haemorrhage, 1 of which in combination with a bowel perforation. This mortality rate is not the mortality of the whole cohort since patients who died within 30 days after transplantation had been excluded (see the Methods section). Frequencies of the clinical outcomes of interest are summarised in table 2.

\section{Gastric retention}

We identified 35 patients $(17.7 \%)$ with radiological evidence of GR, of which almost half were patients suffering from CF. Fifteen patients showed signs of GR without the presence of diabetes. Two of these suffered from CF.

Twenty patients had diabetes and signs of GR. Seventeen of these patients were insulin-dependent. The three patients requiring no insulin were patients with CF.

Associations of various factors with incident GR were explored in unadjusted univariate and in multivariate analyses, and results are presented in table 3 . 
Table 1 Demographics of the study cohort

\begin{tabular}{|c|c|}
\hline Patient characteristics & Number $(\%)^{*}$ \\
\hline All analysed lung transplant recipients & $198(100)$ \\
\hline \multicolumn{2}{|l|}{ Sex } \\
\hline Female, n (\%) & $99(50)$ \\
\hline Male, n (\%) & $99(50)$ \\
\hline \multicolumn{2}{|l|}{ Indication for lung transplantation, n (\%) } \\
\hline Cystic fibrosis (CF) & $78(39.4)$ \\
\hline Chronic obstructive pulmonary disease (COPD) & $52(26.3)$ \\
\hline Idiopathic pulmonary fibrosis (IPF) & $32(16.2)$ \\
\hline Pulmonary arterial hypertension (PAH) & $10(5.1)$ \\
\hline Other & $26(13.1)$ \\
\hline Follow-up time†, median (range) & 5.7 years ( 74 days -12.9 years) \\
\hline Total follow-up time of cohort & 1274 patient-years \\
\hline \multicolumn{2}{|c|}{$\begin{array}{l}\text { Frequency of abdominal plain radiographs per patient, normalised for follow-up time. Categories by mean n APF per } \\
\text { patient-year }\end{array}$} \\
\hline 0 & $33(16.7)$ \\
\hline$>0$ to $\leq 0.5$ & $50(25.3)$ \\
\hline$>0.5$ to $\leq 1$ & 38 (19.2) \\
\hline$>1$ to $\leq 2$ & $44(22.2)$ \\
\hline$>2$ & $33(16.7)$ \\
\hline
\end{tabular}

${ }^{*}$ Results given as number (\%) unless indicated otherwise.

†Follow-up period: From January 2000 (or from date of lung transplantation if transplantation was performed after January 2001) to end of November 2013.

Table 2 Observed outcomes during follow-up of the study cohort

\begin{tabular}{|c|c|}
\hline Patient characteristics & n $(\%)^{\star}$ \\
\hline All analysed lung transplant recipients & $198(100)$ \\
\hline Deaths during follow-up & $26(13.1)$ \\
\hline Patients with an event of GR & $35(17.7)$ \\
\hline $\mathrm{CF}$ & $17(48,5)$ \\
\hline With diabetes & $12(34)$ \\
\hline COPD & 7 (20) \\
\hline With diabetes & $1(3)$ \\
\hline IPF & $6(17)$ \\
\hline Others & $5(14.5)$ \\
\hline With diabetes & $2(6)$ \\
\hline Patients with any lower gastrointestinal event $†$ & 165 (83.3) \\
\hline Small bowel obstruction & $13 \ddagger(7.5)$ \\
\hline Large bowel obstruction & $14(7.1)$ \\
\hline Coprostasis & $124(62.6)$ \\
\hline Diffuse bowel obstruction & $13(6.6)$ \\
\hline Bowel perforation & $1(0.5)$ \\
\hline Patients with up to 3 lower Gl events & $138(69.7)$ \\
\hline Patients with $>3$ repetitive lower $\mathrm{Gl}$ events & 79 (39.9) \\
\hline $\begin{array}{l}\text { Patients with an event of bronchiolitis } \\
\text { obliterans syndrome }\end{array}$ & $52(26.7)$ \\
\hline \multicolumn{2}{|c|}{$\begin{array}{l}{ }^{*} \text { Results given as number (\%) unless indicated otherwise. } \\
\text { †Patients may have more than one different lower Gl event. The } \\
\text { sum of lower Gl events therefore exceeds the number of patients } \\
\text { with any lower gastrointestinal event. } \\
\text { †One patient with ileus as complication. } \\
\text { BOS, bronchiolitis obliterans syndrome; GI, gastrointestinal. }\end{array}$} \\
\hline
\end{tabular}

Table 3 Univariate and multivariate associations of various factors with gastric retention

\begin{tabular}{|c|c|c|}
\hline Factor & $\begin{array}{l}\text { Relative risk } \\
\text { (unadjusted) } \\
\text { (95\% Cl) }\end{array}$ & $\begin{array}{l}\text { OR (adjusted } \text { (a) }^{*} \\
(95 \% \mathrm{Cl})\end{array}$ \\
\hline Female sex & 1.5 (0.8 to 2.8$)$ & 1.8 (0.8 to 4.0$)$ \\
\hline Age $>50$ at LT & $0.8(0.4$ to 1.5$)$ & 1.3 (0.5 to 3.3$)$ \\
\hline Diabetes & 1.9 (1.0 to 3.4$)$ & 2.5 (1.1 to 6.1$)$ \\
\hline Domperidone & 1.0 (0.3 to 2.8$)$ & 1.1 (0.3 to 4.2$)$ \\
\hline Laxatives & 1.0 (0.5 to 2.2$)$ & $0.9(0.4$ to 2.5$)$ \\
\hline Opioids & $1.4(0.6$ to 3.0$)$ & 1.8 (0.6 to 5.3$)$ \\
\hline Macrolide antibiotics & 0.6 (0.3 to 1.1$)$ & 0.5 (0.2 to 1.2$)$ \\
\hline
\end{tabular}

We found a statistically significant association of diabetes (CF and non-CF-related) with GR in the univariate as well as in the adjusted multivariate analyses. Furthermore, adjusted analysis showed non-significant trends towards an increased risk for the development of GR in female patients $(\mathrm{OR}=1.8,95 \%$ CI 0.8 to 4.0$)$ and those with opioid medication $(\mathrm{OR}=1.8,95 \%$ CI 0.6 to 5.3), and there was a trend towards a protective effect of macrolide antibiotics $(\mathrm{OR}=0.5,95 \%$ CI 0.2 to 1.2$)$. 
Figure 1 Time to event analysis shows a progressive risk of developing gastric retention (GR) after lung transplant, with $\sim 30 \%$ of patients developing evidence of GR on abdominal plan films during follow-up.
Time to event analysis for gastric retention

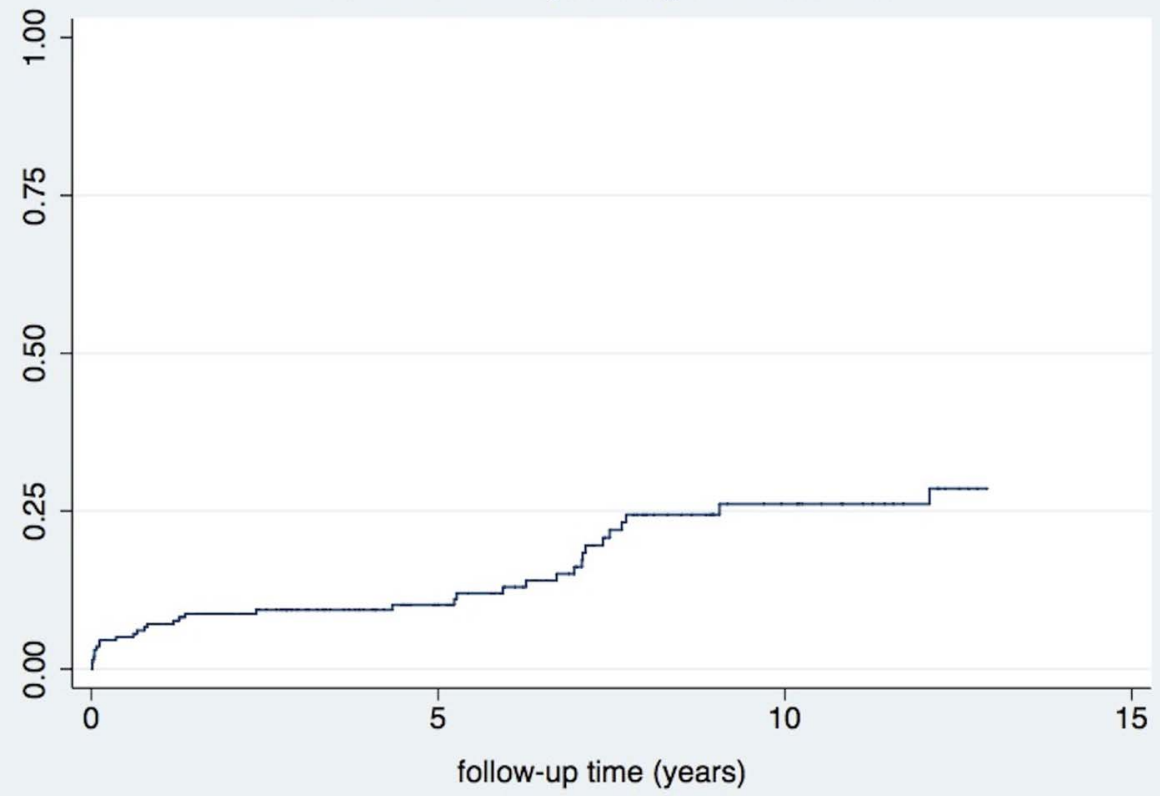

Table 4 Univariate and multivariate associations of various factors with lower gastrointestinal events

\begin{tabular}{lll}
\hline Factor & $\begin{array}{l}\text { Relative risk } \\
\text { (unadjusted) } \\
\text { (95\% CI) }\end{array}$ & $\begin{array}{l}\text { OR (adjusted*) } \\
\text { (95\% CI) }\end{array}$ \\
\hline Female sex & $1.2(1.1$ to 1.4$)$ & $4.4(1.7$ to 11.6$)$ \\
Age $>50$ years at LT & $0.9(0.8$ to 1.1$)$ & $0.8(0.2$ to 2.8$)$ \\
CF† & $1.1(0.9$ to 1.2$)$ & $2.0(0.4$ to 10.5$)$ \\
COPD† & $1.0(0.8$ to 1.1$)$ & $1.1(0.3$ to 4.1$)$ \\
IPF† & $1.0(0.8$ to 1.2$)$ & $1.4(0.3$ to 5.9$)$ \\
PAH† & $1.1(0.9$ to 1.3$)$ & $3.9(0.3$ to 49.9$)$ \\
Diabetes & $1.0(0.9$ to 1.2$)$ & $0.9(0.3$ to 2.6$)$ \\
Domperidone & $1.1(0.8$ to 1.5$)$ & $1.8(0.5$ to 6.9$)$ \\
Laxatives & $1.2(1.0$ to 1.5$)$ & $2.6(1.0$ to 6.5$)$ \\
Opioids & $1.2(1.1$ to 1.3$)$ & $5.2(0.6$ to 41.9$)$ \\
Macrolide antibiotics & $1.0(0.8$ to 1.1$)$ & $0.7(0.3$ to 1.7$)$ \\
\hline *Adjusted from multivariate logistic regression including all listed \\
factors. \\
tlndications for lung transplantation (LT). \\
COPD, chronic obstructive pulmonary disease; IPF, idiopathic \\
pulmonary fibrosis; PAH, pulmonary arterial hypertension.
\end{tabular}

Time to event analysis revealed a progressive risk of developing GR after LT, with $\sim 30 \%$ of patients having evidence of GR on APF 13 years after LT (figure 1).

\section{Lower Gl events}

We identified 165 patients $(83.3 \%)$ with lower GI events. Unadjusted and adjusted associations of various factors with lower GI events are presented in table 4 .

Only one patient needed surgery for ileus and perforation. Further, there was an increased risk for lower GI events associated with female sex, and laxative and opioid use. Of note, due to the high proportion of patients with lower GI events as the outcome of interest, ORs from logistic regression are much higher than the corresponding relative risks. Time to event analysis demonstrated that a high proportion of patients already had evidence of lower GI events early after transplantation and that virtually all patients have evidence of lower GI events when considering long-term follow-up after transplantation (figure 2).

\section{Bronchiolitis obliterans syndrome}

Fifty-two patients $(26.3 \%)$ had evidence of CLAD/BOS based on pulmonary function testing 6 months after the time of the lower GI event. Trends for mildly elevated risks were observed for female sex $(\mathrm{OR}=1.6,95 \%$ CI 0.8 to 3.1), patients who had received transplants for $\mathrm{CF}$ $(\mathrm{OR}=1.5,95 \%$ CI 0.5 to 4.8$)$ and in patients with an event of GR (OR=1.8, 95\% CI 0.8 to 4.0). However, there was no trend towards a higher adjusted risk in patients with more than three lower GI events $(\mathrm{OR}=0.9$, $95 \%$ CI 0.4 to 1.7$)$.

\section{DISCUSSION}

This study assessed the prevalence and possible risk factors for GI dysmotility seen on APF after LT over a median follow-up time of 5.7 years. We found a statistically significant association of diabetes with GR and a prevalence of upper and lower GI complications as documented on clinically indicated APF. Diabetes (type II and CF-related) was identified as an important risk factor for the development of GR in patients after LT. Furthermore, the risk of developing GR increases with time after transplantation. For lower GI complications such as coprostasis, more than $80 \%$ of patients suffered at least one episode after LT, and similar to GR, the prevalence of lower GI events increases with time after transplantation. 
Figure 2 A high proportion of patients' experiences lower gastrointestinal events shortly after transplantation and that virtually all patients accumulate evidence of a lower gastrointestinal event during follow-up time after transplantation.

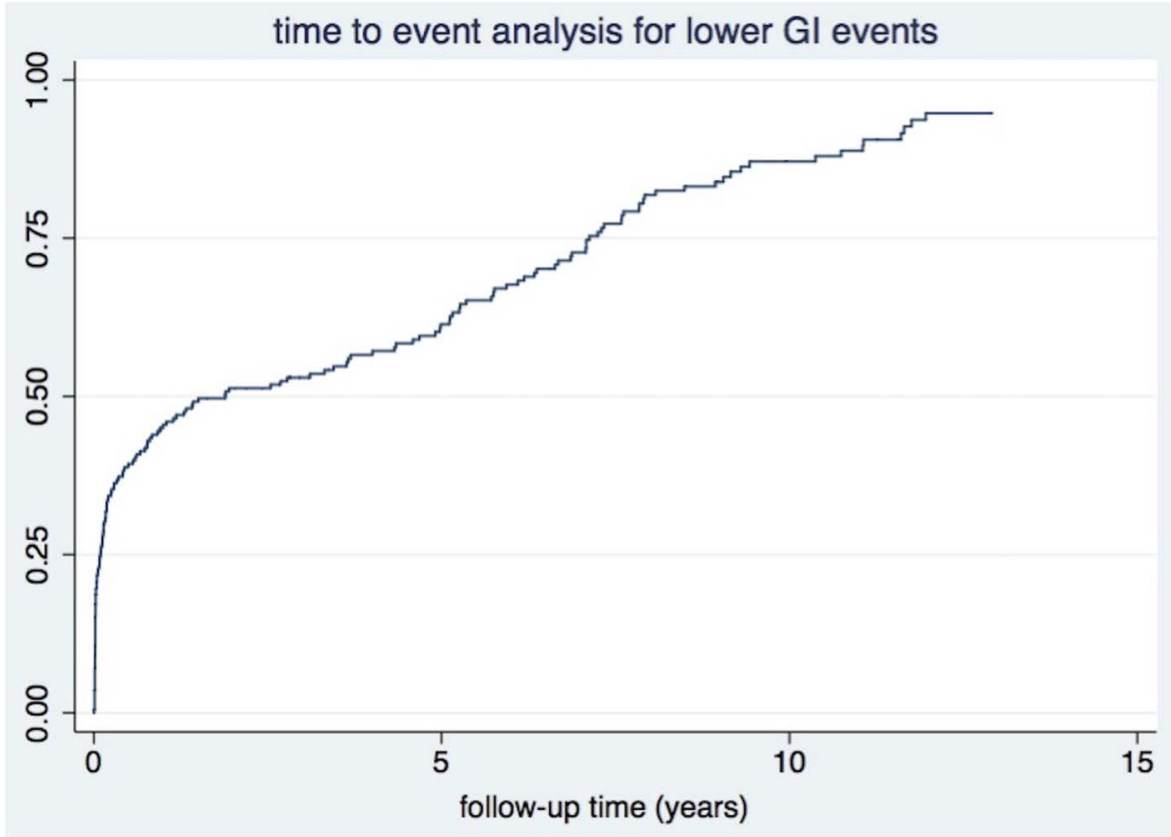

The reported prevalence of upper and lower GI complications after LT including malignancy is highly variable in the literature depending on definition of complications, study focus and study population. ${ }^{8-15}$ A recent retrospective study over 17 years reported $10.7 \%$ of patients suffering from gastroparesis after LT using a clinical definition. ${ }^{8}$ In our study population, $17.7 \%$ of patients showed evidence of GR on APF reflecting the larger proportion of patients with $\mathrm{CF}$ and diabetes in our cohort. Whether the presence of CF-related diabetes and diabetes mellitus in general have impact on survival after transplantation is disputed in the literature. ${ }^{16} 17$ Multivariate regression showed a significant correlation between the presence of diabetes and GR. This pathophysiologically plausible result suggests that diabetes is a causal contributor to the development of GR in LTRs. Vagal nerve damage is frequently mentioned as an important reason for gastroparesis after LT surgery; ${ }^{3}$ however, the low prevalence of GR within the first 30 days after LT somewhat argues against this mechanism as the predominant explanation for GR in most patients.

In addition, our results suggest that other causes than diabetes such as medication related to LT play a role in the development of GR and occurred despite standard prokinetic treatment in all patients. Furthermore, GR did not exclusively manifest in CF patients with diabetes, but also in non-CF patients with and without diabetes, suggesting a multifactorial mechanism for the development of GR in LTRs. GR with possible gastrooesophageal reflux and aspiration poses a significant risk for the development of BOS. ${ }^{18}{ }^{19}$ The prevalence of GR increased progressively in our study population, with about $30 \%$ of patients having had an episode of GR during an observation time of up to 13 years after transplantation. In our study population, we were able to show that more than $25 \%$ of patients in our study cohort with diabetes and GR fulfilled criteria of BOS 6 months after an upper GI event. Although this did not reach statistical significance, this finding emphasises the importance of adequate medical diabetes management pretransplantation and post-transplantation, and initiating prompt additional prokinetic treatment should GR develop.

Based on published literature and our own clinical observations, we assume that practically all LTRs have gastro-oesophageal reflux to some extent, and therefore, we implement physical, dietary and pharmacological antireflux measures for all patients prophylactically. ${ }^{5}$ In our cohort, none of the patients underwent antireflux surgery. Future studies should investigate the utility of routine gastric emptying and reflux testing in patients after LT as a prognostic tool to tailor prevention and treatment regarding the high percentage of GR in the LT population.

Over $80 \%$ of patients in our study showed evidence of impaired lower GI motility on APF during follow-up, with coprostasis being the most common finding. Most studies report an incidence of around $40 \%$ regarding colitis and diverticulitis after LT. ${ }^{8}$ The high proportion of patients with lower GI dysmotility detected in our study can be attributed to mostly minor medical complications like coprostasis not being assessed in most other studies and the low threshold for diagnostic tests in the setting of abdominal symptoms at our centre. In our practice, laxative therapy is intensified in patients after LT when coprostasis is seen on APF to minimise the risk of abdominal complications such as vomiting, diverticulitis or perforation.

Furthermore, our results demonstrate that virtually all patients suffer from impaired lower GI motility after LT at some point in time in spite of standard laxative therapy, and that the prevalence increases with time 
after transplantation, suggesting a late-onset aetiology such as autonomic neuropathy or possible vascular changes that may develop many years after LT.

Opioid medication should be used with caution in patients after LT, mainly due to its slowing effect on GI motility. Our data show that patients who received opioid medication had a trend towards a higher risk of developing upper or lower GI complications like GR or colonic motility impairment. Possibly due to the sparse administration of opioids at our centre, this trend failed to reach statistical significance and only one patient required surgery due to ileus and bowel perforation. ${ }^{6}$

Our study has several limitations. Data were collected retrospectively, which makes the data vulnerable to bias related to staff turnover and evolution of medical practice and technology over time. APFs were performed at the discretion of the treating physician, which might contribute to a selection bias. However, the threshold for performing an APF in LT patients presenting with abdominal symptoms has been consistently low at our centre over time. Our data comprise a large number of LTR who were treated according to standardised procedures and with objective measurements, so that data of prescribed medication, lung function and treatment modifications are of high quality. A further limitation is the lack of verification of gastroparesis by gastric emptying studies such as 13C breath tests, which however also have inherent limitations. The sensitivity and specificity of APF for GR could therefore not be assessed. In addition, even though all radiographs were evaluated by our study team with discussion of ambiguous cases, a formal analysis for interobserver agreement was not performed. Furthermore, clinical decision-making is a complex process and our chart review could not unequivocally identify changes in management caused by APF.

Although no direct comparison of APF and CT scan results was made, the low incidence of major GI complications as assessed by the need for surgical intervention in our cohort when compared with other cohorts may suggest that the strategy of using APF as a primary diagnostic tool besides obtaining routine laboratory investigations might be considered useful and help justify its utility in clinical practice. ${ }^{6}$ Cost, radiation exposure, time and availability are other factors that favour our approach using an APF as an initial diagnostic tool in LT patients with unspecific abdominal symptoms.

Lower and upper GI complications are common and have been described previously after LT. APF can easily and swiftly be obtained and can provide useful information: APF consistent with coprostasis or GR as an explanation for patient symptoms would prompt intensified medical treatment or aggressive diagnostics such as CT scan in the case of the presence of bowel obstruction or signs of perforation. Follow-up APF also provides an objective measure for resolution of coporostasis and GR in the case of ambiguous clinical findings.

The results of our study have important implications for clinical practice: Underlying medical conditions like diabetes are a clinically important risk for the development of GR and opioid medication should be avoided whenever possible after LT. A high level of suspicion is warranted concerning the development of coprostasis despite laxative treatment. AFP provides quick information if GI dysmotility is present and can stratify the need for further diagnostics such as computer tomography and can guide intensified prokinetic and laxative treatment. Further studies should evaluate whether a first event of gastric/ intestinal dysmotility after LT should trigger further motility testing like gastric emptying studies, $\mathrm{pH}$ metry for reflux testing and intestinal transit studies, whether these tests identify risk groups for GI dysmotility and if the results have an impact on treatment, outcome and survival. While management of LT patients should ideally be carried out at specialised centres, we recommend to plan treatment in a multidisciplinary team, which should involve gastroenterologists, in particular motility specialists when recurrent episodes of GI dysmotility are noted.

Contributors HH is responsible for concept/design, data analysis/ interpretation, drafting article, critical revision of article and approval of article. AN is responsible for data analysis/interpretation, drafting article and data collection. SR is responsible for data analysis/interpretation and critical revision of article. BM is responsible for critical revision of article and approval of article. CB is responsible for critical revision of article and approval of article. MMS is responsible for concept/design, data analysis/ interpretation, drafting article, critical revision of article and approval of article.

Funding This study received no funding but was supported by the Division of Pulmonary Medicine and Division of Gastroenterology and Hepatology, respectively.

Competing interests None declared.

Ethics approval Zuerich Ethics comittee.

Provenance and peer review Not commissioned; externally peer reviewed.

Data sharing statement No additional data are available.

Open Access This is an Open Access article distributed in accordance with the Creative Commons Attribution Non Commercial (CC BY-NC 4.0) license, which permits others to distribute, remix, adapt, build upon this work noncommercially, and license their derivative works on different terms, provided the original work is properly cited and the use is non-commercial. See: http:// creativecommons.org/licenses/by-nc/4.0/

\section{REFERENCES}

1. Fuehner $\mathrm{T}$, Welte $\mathrm{T}$, Simon $\mathrm{A}$, et al. [Complications after lung transplantation: gastroenterological, hematological and cardiovascular involvement]. Dtsch Med Wochenschr 2008;133:836-9.

2. Miller CB, Malaisrie SC, Patel J, et al. Intraabdominal complications after lung transplantation. J Am Coll Surg 2006;203:653-60.

3. Gautam A. Gastrointestinal complications following transplantation. Surg Clin North Am 2006;86:1195-206. vii.

4. Timrott $\mathrm{K}$, Vondran FW, Jaeger M, et al. Incidence and outcome of abdominal surgical interventions following lung transplantation-a single center experience. Langenbecks Arch Surg 2011;396:1231-7.

5. Schuurmans MM, Tini GM, Zuercher A, et al. Practical approach to emergencies in lung transplant recipients: how we do it. Respiration 2012;84:163-75.

6. Vetter D, Schuurmans MM, Benden C, et al. Long-term follow-up of lung transplant recipients supports non-operative treatment of uncomplicated diverticulitis. Clin Transplant 2016;30:1264-70.

7. Timrott K, Vondran FWR, Kleine M, et al. The impact of abdominal complications on the outcome after thoracic transplantation-a single center experience. Langenbecks Arch Surg 2014;399:789-93.

8. Grass F, Schäfer M, Cristaudi A, et al. Incidence and risk factors of abdominal complications after lung transplantation. World J Surg 2015;39:2274-81. 
9. Musson RE, Bickle I, Vijay RKP. Gas patterns on plain abdominal radiographs: a pictorial review. Postgrad Med J 2011;87:274-87.

10. James B, Kelly B. The abdominal radiograph. Ulster Med J 2013;82:179-87.

11. Park HJ, Noh SE, Kim GD, et al. Plain abdominal radiograph as an evaluation method of bowel dysfunction in patients with spinal cord injury. Ann Rehabil Med 2013;37:547-55.

12. Meyer KC, Raghu G, Verleden GM, et al. An international ISHLT/ ATS/ERS clinical practice guideline: diagnosis and management of bronchiolitis obliterans syndrome. Eur Respir J 2014;44:1479-503.

13. Lubetkin El, Lipson DA, Palevsky HI, et al. Gl complications after orthotopic lung transplantation. Am J Gastroenterol 1996;91:2382-90.

14. Bravo C, Gispert P, Borro JM, et al. Prevalence and management of gastrointestinal complications in lung transplant patients: MITOS study group. Transplant Proc 2007;39:2409-12.
15. Murer C, Bürgi U, Kohler M, et al. Adenocarcinoma of the gastrointestinal tract in lung transplanted patients with cystic fibrosis: case series and review of the literature. Swiss Med Wkly 2015;145: w14165.

16. Hofer M, Benden C, Inci I, et al. True survival benefit of lung transplantation for cystic fibrosis patients: the Zurich experience. $J$ Heart Lung Transplant 2009;28:334-9.

17. Moreno $P$, Alvarez A, Carrasco G, et al. Lung transplantation for cystic fibrosis: differential characteristics and outcomes between children and adults. Eur J Cardiothorac Surg 2016;49:1334-43.

18. Fisichella PM, Davis CS, Kovacs EJ. A review of the role of GERD-induced aspiration after lung transplantation. Surg Endosc 2012;26:1201-4.

19. Fisichella PM, Jalilvand A. The role of impaired esophageal and gastric motility in end-stage lung diseases and after lung transplantation. J Surg Res 2014;186:201-6. 